\title{
Atividade antioxidante de tinturas vegetais, vendidas em farmácias com manipulação e indicadas para diversos tipos de doenças pela metodologia do DPPH
}

\author{
Amanda R. R. Vicentino, Fábio de Sousa Menezes* \\ Departamento de Produtos Naturais e Alimentos, Faculdade de Farmácia, Centro de Ciências da Saúde, \\ Universidade Federal do Rio de Janeiro, 21941-590, Rio de Janeiro, RJ, Brasil
}

\begin{abstract}
RESUMO: O presente trabalho descreve a avaliação da atividade antioxidante pela metodologia do radical livre DPPH de tinturas comerciais obtidas de farmácias com manipulação indicadas para os mais variados tipos de doença que apresentam em maior ou menor grau, alguma etiologia fundamentada em excesso de radicais livres. Foi interessante notar uma sincronia entre a família a que a planta de determinada tintura pertence, com suas substâncias químicas e a atividade antioxidante esperada. Para os casos em que isso não ocorreu, pode-se verificar através de métodos cromatográficos que as tinturas não haviam sido preparadas de forma adequada ou se tratava de falsificações.
\end{abstract}

Unitermos: Atividade antioxidante, tinturas comercias, composição química, falsificações.

\begin{abstract}
Antioxidant activity of plant tinctures, commonly sold in pharmacies and indicated for several types of diseases, using the DPPH methodology". This work describes the antioxidant evaluation through the DPPH free radical methodology of commercial available tinctures indicated for different diseases and purchased in commercial pharmacies. All of those diseases have always being caused by excess or accumulation of free radicals. It was noteworthy the coherence between the botany family in which the plant of each tincture is inserted as well as its chemical composition with the antioxidant activity observed. In the cases where this statement could not be shown, it was proved by chromatographic methods that the tinctures were not properly prepared or even it constituted a case of adulteration.
\end{abstract}

Keywords: Antioxidant activity, commercial tinctures, chemical compositions, adulterations.

\section{INTRODUÇÃO}

Exceto em microrganismos anaeróbicos, em todos os sistemas biológicos, desde os mais simples, como os procariontes até os mais especializados como os mamíferos e plantas (Sorg, 2004) as espécies reativas de oxigênio (ERO) são produzidas nas mitocôndrias como um subproduto do metabolismo normal (Fridivitch, 1999) durante a produção de energia (Sorg, 2004). As ERO exercem ações na tradução, na transcrição gênica e na regulação da atividade da guanilato ciclase nas células (Zheng; Storz, 2000).

Os exemplos de ERO mais comuns são os radicais superóxido $\left(\mathrm{O}_{2}^{-}\right)$, hidroxila $(\mathrm{OH})$, peroxila $\left(\mathrm{RO}_{2}\right)$, alkoxila (RO) e hidroperoxila $\left(\mathrm{HO}_{2}\right)$. O óxido nítrico (NO) e o dióxido de nitrogênio $\left(\mathrm{NO}_{2}\right)$ são espécies reativas de nitrogênio (Souza et al., 2007). Outras moléculas como o peróxido de hidrogênio $\left(\mathrm{H}_{2} \mathrm{O}_{2}\right)$ e o peroxinitrato (ONOO) não são radicais livres, mas podem conduzir à formação deles através de reações químicas (Evans; Halliwell, 2001).

O estresse oxidativo (EO) corresponde a um desequilíbrio entre a taxa de produção de agentes oxidantes e sua degradação (Sies, 1991). Ocorre quando a produção de ERO está acelerada ou quando os mecanismos envolvidos na proteção contra as ERO encontram-se deteriorados (Giasson et al., 2002). Predisposição genética, fatores ambientais como radiação UV e propriedades intrínsecas específicas de grupos celulares podem exacerbar o dano oxidativo ou diminuir a capacidade das células de degradar estes agentes agressores (Giasson et al., 2002).

A peroxidação lipídica é a principal conseqüência do estresse oxidativo resultando na danificação das membranas causada pela oxidação acarretando em aumento da fluidez da membrana, comprometimento da sua integridade e inativação da interação entre membrana/receptor e membrana/enzima (Halliwell; Gutterridge, 1986).

Acredita-se que as doenças degenerativas crônicas como doença de Alzheimer, doença de Parkinson, aterosclerose, complicações da Diabetes mellitus, o envelhecimento precoce e outras estejam relacionadas com o estresse oxidativo (Sorg, 2004).

Os antioxidantes são um grupo de substâncias que, quando presentes em concentrações ideais em 
relação aos substratos oxidáveis, inibem ou atrasam significativamente os processos oxidativos podendo ser divididos em enzimáticos, solúveis, nutricionais e seqüestradores de metais de transição (Vaya; Aviram, 2001).

Esse trabalho propõe dosar a atividade antioxidante pela metodologia do radical livre estável DPPH, das tinturas-mães comercializadas por farmácias de manipulação e farmácias homeopáticas com o intuito de verificar se as mesmas possuem alguma atividade antioxidante, o que poderia caracterizar um dos mecanismos de ação que explicariam as suas diferentes utilizações, uma vez que se sabe que cerca de $95 \%$ das doenças degenerativas crônicas são induzidas por estresse oxidativo e muitas dessas tinturas seriam utilizadas diretamente para combater essas doenças ou indiretamente como preventivo das mesmas.

\section{MATERIAL E MÉTODOS}

\section{Material vegetal}

As tinturas-mães de Camomila (Matricaria chamomilla $\quad$ L.), Castanha-da-índia (Aesculus hippocastanum L.), Centela asiática (Hydrocotile asiatica L.), Erva cidreira (Melissa officinalis L.), Ginkgo (Ginkgo biloba L.), Goiabeira (Psidium guajava L.), Guaco (Mikania glomerata), Hamamelis (Hamamelis virginiana L.), Jaborandi (Pilocarpus jaborandi Hulmes), Mulungu (Erythrina velutina Wild), Passiflora (Passiflora coerulea L.), Pimentão (Capsicum annuum L.), Poejo (Mentha pulegium L.) e Valeriana (Valeriana officinallis L.), além de Própolis foram adquiridas de uma farmácia homeopática e evaporadas sob pressão reduzida à temperatura inferior a $50^{\circ} \mathrm{C}$ a fim de se obter o material seco para cada espécie.

\section{Dosagem da atividade antioxidante}

A dosagem de atividade antioxidante foi realizada pelo método fotocolorimétrico in vitro do radical livre estável DPPH (2,2-difenil-1-picrilidrazila) obtido da SIGMA. Nesse método é preparada uma solução 0,3 mM de DPPH em etanol. As amostras são preparadas adicionando-se $1 \mathrm{~mL}$ desta solução de DPPH a 2,5 mL de soluções dos extratos diluídos em etanol a concentrações de $5,10,25,50,125$ e $250 \mu \mathrm{g} / \mathrm{mL}$. Nos brancos, ao invés do DPPH, adiciona-se apenas $1 \mathrm{~mL}$ de etanol aos extratos diluídos nas mesmas concentrações das amostras. O controle negativo é preparado apenas com $1 \mathrm{~mL}$ de DPPH e 2,5 mL de etanol. Como controle positivo foi utilizado o extrato padronizado de Ginkgo biloba, EGb $761^{\circledR}$, que possui alta capacidade antioxidante.

A solução de DPPH possui uma coloração roxa intensa e a ação antioxidante de um extrato pode ser visualizada pelo progressivo descoloramento da solução, ao final do qual a mesma torna-se amarelada. Trinta minutos após a adição de DPPH às amostras, é feita a leitura em um espectrofotômetro de Ultravioleta UV-vis Shimadzu UV $1601 \mathrm{em} 518 \mathrm{~nm}$. Todas as leituras foram realizadas em triplicata e, com a média dos dados obtidos foi calculada a diferença de absorbância entre a amostra e o branco e as atividades antioxidantes percentuais foram obtidas por regressão linear, logarítmica ou exponencial para cada extrato, chegandose assim à concentração necessária para se obter $50 \%$ do efeito antioxidante máximo estimado de $100 \%\left(\mathrm{CE}_{50}\right)$ (Mensor et al., 2001).

\section{RESULTADOS E DISCUSSÃO}

A análise dos resultados desse trabalho considera como valor de referência a $\mathrm{CE}_{50}$ do Ginkgo biloba $(38,91 \mu \mathrm{g} / \mathrm{mL})$ para comparar a atividade antioxidante das outras espécies estudadas. Foi escolhida a planta G. biloba como parâmetro porque ela é uma das plantas consideradas com alta atividade antioxidante (Mensor et al., 2001).

A partir dos resultados apresentados na Tabela 1 pode-se observar que a tintura de Hamamelis virginiana quando comparada com a da $G$. biloba possui uma $\mathrm{CE}_{50}$ igual a $0,13 \mu \mathrm{g} / \mathrm{mL}$, isso indica que esta planta possui uma atividade antioxidante (AAO) cerca de 300 vezes maior que a apresentada pelo padrão $G$. biloba. Esse fato se deve provavelmente ao alto teor de taninos presentes nessa espécie, com variados tipos estruturais (Pereira da Silva et al., 2000).

As tinturas de Psidium guajava e de G. biloba apresentam praticamente a mesma $\mathrm{CE}_{50}$ com o mesmo potencial de AAO. Isso pode ser explicado devido às altas concentrações de taninos presentes na $P$. guajava que é pertencente à família Myrtaceae, provavelmente com diferenças estruturais em relação aos taninos presentes na tintura de Hamamelis virginiana (Rahmat et al., 2004).

A tintura de própolis também apresentou uma AAO bem próxima da tintura de G. biloba. Isso se deve ao fato de que a própolis, material resinoso elaborado pelas abelhas a partir de brotos, cascas e outras partes do tecido vegetal de árvores e plantas, de um modo geral, é constituída principalmente por flavonóides, ácidos aromáticos e seus derivados (Russo et al., 2004; Sousa et al., 2007).

A tintura de Matricaria chamomilla apresentou uma $\mathrm{CE}_{50}$ igual a $78,22 \mu \mathrm{g} / \mathrm{mL}$ representando também uma boa AAO apesar de ser duas vezes menos potente que a tintura de G. biloba. A Camomila pertence à família Asteraceae e nela estão presentes substâncias como lactonas sesquiterpênicas, flavonóides e alguns taninos que contribuem para o caráter antioxidante observado (Avallone et al., 2000).

A tintura de Hydrocotile asiatica apresentou uma $\mathrm{CE}_{50}$ igual a $87,61 \mu \mathrm{g} / \mathrm{mL}$ sendo aproximadamente, 
sua AAO duas vezes menor que a tintura de G. biloba. Essa AAO observada se deve a presença de flavonóides em sua constituição (Jaswir, 2004).

As tinturas de Mentha pullegium e Aesculus hippocastanum apresentaram respectivamente uma $\mathrm{CE}_{50}$ igual a 155,04 e $165,0 \mu \mathrm{g} / \mathrm{mL}$ demonstrando ainda alguma AAO apesar de serem aproximadamente quatro vezes menor que a apresentada pela tintura de G. biloba. Isso se deve a baixa concentração de flavonóides na composição química de M. pullegium (Mensor et al., 2001). No caso da tintura de $A$. hippocastanum, esse valor não foi satisfatório quando se sabe que a constituição química da planta é de taninos catéquicos, heterosídeos cumarínicos e derivados flavônicos, sugerindo que o método fotocolorimétrico do DPPH nesse caso não pode ser eficaz porque depende da estrutura conformacional da substância para doar hidrogênio à molécula de DPPH e talvez as substâncias da Castanha-da-índia não tenham boa conformação capaz de estabilizar o radical (Nishizawa et al., 2005; Martins; Brandão, 2006).

A tintura de Erythrina velutina já demonstra baixa AAO quando comparado com a tintura de $G$. biloba, cerca de cinco vezes. A tintura de Passiflora apresentou uma $\mathrm{CE}_{50}$ igual a $245,84 \mu \mathrm{g} / \mathrm{mL}$ o que representa uma AAO seis vezes menor que a tintura de G. biloba. A tintura de Pilocarpus jaborandi demonstrou AAO cerca de onze vezes menor que a da G. biloba. A tintura de Melissa officinallis apresentou AAO baixa. Sua $\mathrm{CE}_{50}$ é igual a $463,29 \mu \mathrm{g} / \mathrm{mL}$ e isso indica que a tintura de $G$. biloba é aproximadamente doze vezes mais potente que a tintura de Melissa. A tintura de Capsicum annum apresentou cerca de vinte e três vezes menos atividade antioxidante que a tintura de G. biloba, ou seja, praticamente nenhuma AAO nesse modelo assim como a tintura de Mikania glomerata que apresentou AAO cerca de trinta e três vezes menor que a da $G$. biloba. Essas espécies apresentam baixa ou nenhuma concentração de substâncias com potencial antioxidante (Mensor et al., 2001; (Taleb-Contini et al., 2006) A realização de procedimentos cromatográficos indicou que as tinturas dessas espécies foram preparadas de forma não adequada.

\section{CONCLUSÃO}

Nesse trabalho, as tinturas de Hamamelis virginiana, Psidium guajava, Própolis, Matricaria chamomilla e Hydrocotile asiatica apresentaram alta atividade antioxidante pelo método fotocolorimétrico do DPPH. As demais tinturas que não apresentaram boa atividade antioxidante por esse método deverão sofrer outros testes para avaliar suas atividades antioxidantes tais como testes bioquímicos como o de peroxidação lipídica, o de captura de radical aniôn superóxido, o de danos à desoxirribose dentre outros para comprovar as atividades antioxidantes. Além disso, ficou comprovado por métodos cromatográficos que algumas tinturas foram preparadas de forma inadequada ou se tratava realmente de falsificações, pois não foi observada a presença de substâncias características da planta, como por exemplo,

Tabela 1. Atividade antioxidante $(\%)^{*}$ das espécies vegetais e própolis testadas pela metodologia do radical livre estável DPPH e determinação da $\mathrm{CE}_{50}$.

\begin{tabular}{lccccccc}
\hline Espécies Estudadas & $5 \mu \mathrm{g} / \mathrm{mL}$ & $10 \mu \mathrm{g} / \mathrm{mL}$ & $25 \mu \mathrm{g} / \mathrm{mL}$ & $50 \mu \mathrm{g} / \mathrm{mL}$ & $125 \mu \mathrm{g} / \mathrm{mL}$ & $250 \mu \mathrm{g} / \mathrm{mL}$ & $\mathrm{CE}_{50}{ }^{* *}$ \\
\hline Controle & $4,57 \pm 1,51$ & $10,03 \pm 3,25$ & $28,14 \pm 1,37$ & $57,02 \pm 0,55$ & $90,07 \pm 0,82$ & $95,23 \pm 0,43$ & 38,91 \\
H. virginiana & $60,69 \pm 1,79$ & $84,63 \pm 0,22$ & $85,88 \pm 0,34$ & $88,36 \pm 0,07$ & $91,69 \pm 0,63$ & $94,07 \pm 0,36$ & 0,13 \\
P. guajava & $0,52 \pm 0,85$ & $14,28 \pm 5,07$ & $46,26 \pm 2,89$ & $82,99 \pm 0,52$ & $92,2 \pm 0,62$ & $97,35 \pm 0,41$ & 33,57 \\
Própolis & $3,27 \pm 1,17$ & $8,60 \pm 0,66$ & $21,02 \pm 1,47$ & $39,55 \pm 1,53$ & $74,51 \pm 1,48$ & $90,76 \pm 0,07$ & 40,16 \\
M. chamomilla & $22,76 \pm 1,60$ & $25,95 \pm 1,79$ & $34,93 \pm 1,50$ & $45,10 \pm 0,63$ & $77,52 \pm 1,86$ & $92,49 \pm 0,51$ & 78,22 \\
H. asiatica & $0,45 \pm 4,05$ & $1,25 \pm 4,87$ & $8,24 \pm 3,91$ & $24,09 \pm 2,76$ & $62,92 \pm 2,04$ & $82,99 \pm 2,43$ & 87,61 \\
M. pullegium & $0,01 \pm 3,85$ & $0,68 \pm 1,43$ & $6,56 \pm 2,50$ & $11,43 \pm 1,11$ & $29,52 \pm 0,42$ & $88,31 \pm 7,03$ & 155,04 \\
A. hippocastanum & $2,72 \pm 0,24$ & $3,66 \pm 0,94$ & $8,67 \pm 0,95$ & $16,83 \pm 0,74$ & $39,49 \pm 1,91$ & $73,82 \pm 1,84$ & 165,80 \\
E. velutina & $0,17 \pm 3,44$ & $4,32 \pm 1,07$ & $7,71 \pm 0,68$ & $15,32 \pm 1,72$ & $35,18 \pm 0,88$ & $57,57 \pm 1,72$ & 207,15 \\
P. coerula & $3,43 \pm 1,30$ & $5,89 \pm 1,09$ & $8,22 \pm 1,94$ & $12,06 \pm 1,41$ & $26,02 \pm 1,69$ & $51,26 \pm 3,82$ & 245,84 \\
V. officinalis & $0,23 \pm 1,02$ & $1,55 \pm 0,72$ & $4,94 \pm 0,90$ & $9,35 \pm 2,15$ & $24,86 \pm 1,50$ & $42,74 \pm 2,19$ & 283,63 \\
P. jaborandi & $1,90 \pm 0,71$ & $1,92 \pm 0,21$ & $6,93 \pm 1,33$ & $8,33 \pm 0,42$ & $16,92 \pm 1,10$ & $29,71 \pm 1,14$ & 429,30 \\
M. officinalis & $0,39 \pm 0,98$ & $0,51 \pm 2,04$, & $1,23 \pm 7,68$ & $5,73 \pm 1,62$ & $13,74 \pm 1,29$ & $25,86 \pm 0,30$ & 463,29 \\
C. annum & $0,41 \pm 0,82$ & $0,52 \pm 0,83$ & $3,18 \pm 0,81$ & $4,40 \pm 1,81$ & $8,53 \pm 1,58$ & $13,57 \pm 2,17$ & 906,08 \\
M. glomerata & $5,92 \pm 1,36$ & $6,77 \pm 1,16$ & $6,59 \pm 0,68$ & $8,71 \pm 1,41$ & $11,06 \pm 1,63$ & $14,37 \pm 1,59$ & 1283,88 \\
\hline
\end{tabular}

Controle positivo: extrato padronizado de Ginkgo biloba $\left(\mathrm{EGb} 761^{\circledR}\right)$ nas mesmas concentrações que os extratos.

*\% de atividade antioxidante para cada uma das concentrações após a média de três análises e aplicação da seguinte fórmula:

$\%$ Atividade Antioxidante $(\% \mathrm{AA})=100-(\triangle \mathrm{ABS} \times 100) / \mathrm{ABS}_{\text {cont }}$ onde: $\triangle \mathrm{ABS}$ é a diferença de absorbância entre a amostra e o branco e $\mathrm{ABS}_{\text {cont }}$ é o valor de absorbância encontrado para o controle ${ }^{* *}$ Concentração efetiva $50 \%$ calculada através de regressão linear, logarítmica ou exponencial do gráfico obtido com os valores encontrados de \%AA para cada concentração. 
a capsaicina no caso da tintura de Capsicum.

Esse estudo colabora, portanto, para o conhecimento da atividade antioxidante pelo método do DPPH de algumas espécies vegetais que de forma popular no Brasil são muito utilizadas no tratamento de diversas doenças com etiologia em distúrbios oxidativos, sem nenhuma comprovação científica.

\section{REFERÊNCIAS}

Avallone R, Zanoli P, Puia G, Kleinschnitz M, Schereier P, Baraldi M 2000. Pharmacological profile of apigenin, a flavonoid isolates from Matricaria chamomilla. Phytother Res 14: 612-616.

Evans P, Halliwell B 2001. Micronutrients: oxidant/antioxidant status. Brit J Nutr 85 (Suppl 2): S67.

Fridivitch I 1999. Fundamental aspects of reactive oxygen species, or what's the matter with oxygen? Ann NY Acad Sci 13: 893.

Giasson BI, Ischiropoulos H, Lee VMY, Trojanowski JQ 2002. The relationship between oxidative/nitrative stress and pathological inclusions in Alzheimer's and Parkison's diseases. Free Radical Bio Med 32: 1264-1275.

Halliwell B, Gutteridge JMC 1986. Oxygen free radicals and iron in relation to biology and medicine: some problems and concepts. Arch Biochem Biophys 246: 501-514.

Jaswie I, Hassan TH, Said MZ 2004. Antioxidative behaviour of Malaysian plant extracts in model and food oil systems. Asia Pac J Clin Nutr 13(Suppl): S72.

Martins ELP, Brandão MGL 2006. Qualidade de amostras comerciais preparadas com Aesculus hippocastanum L. (Castanha-da-Índia). Rev Bras Farmacogn 16: 224-229.

Mensor LL, Menezes FS, Leitão GG, Reis AS, dos Santos TC, Coube CS, Leitão SG 2001. Screnning of Brazilian plant extracts for antioxidant activity by the use of DPPH free radical method. Phytother Res 15: 127130.

Nishizawa M, Kohno M, Nishimura M, Kitagawa, A, Niwano $Y$ 2005. Non-reductive scavenging of 1,1-diphenyl2-picrylhydrazyl (DPPH) by peroxyradical: a useful method for quantitative analysis of peroxyradical. Chem Pharm Bull 53: 714-716.

Pereira da Silva A, Rocha R, Silva CM, Mira L, Duarte MF, Florencio MH 2000. Antioxidants in medicinal plant extracts. A research study of the antioxidant capacity of Crataegus, Hamamelis and Hydrastis. Phytother Res 14: 612-616.

Rahmat A, Abu Bakar MF, Faezah N, Hambali Z 2004. The effects of consumption of guava (Psidium guajava) or papaya (Carica papaya) on total antioxidant and lipid profile in normal male youth. Asia Pac J Clin Nutr 13: 106-111.

Russo A, Cardile V, Sanchez F, Troncoso N, Vanella A, Garbarino JA 2004. Chilean própolis: antioxidant activity and antiproloferative action in human tumor cells lines. Life Sci 76: 545-558.

Sies H 1991. Oxidative stress: from basic research to clinical application. Am J Med 91 (Suppl 3C): 31S-38S.

Sorg O 2004. Oxidative stress: a theoretical model or biological reality? $C R$ Biol 327: 649-662.

Sousa JPB, Furtado NAJC, Jorge R, Soares AEE, Bastos JK 2007. Perfis físico-químico e cromatográfico de amostras de própolis produzidas nas microrregiões de Franca (SP) e Passos (MG), Brasil. Rev Bras Farmacogn 17: 85-93.

Souza WM, Brehmer F, Nakao LS, Stinghen AEM, Santos CAM 2007. Ação da uleína sobre a produção de óxido nítrico em células RAEC e B16F10. Rev Bras Farmacogn 17: 191-196.

Taleb-Contini SH, Santos PA, Veneziani RCS, Pereira AMS, França SC, Lopes NP, C.R. Oliveira DCR 2006. Differences in secondary metabolites from leaf extracts of Mikania glomerata Sprengel obtained by micropropagation and cuttings. Rev Bras Farmacogn 16: 596-598.

Vaya J, Aviram M 2001. Nutritional antioxidants: mechanisms of action, analyses if activities and medical applications. Curr Med Chem Imm Endoc Metab Agents 1: 99-117.

Zheng M, Storz G 2000. Redox sensing by prokaryotic transcription factors. Biochem Pharmacol 59: 1-6.

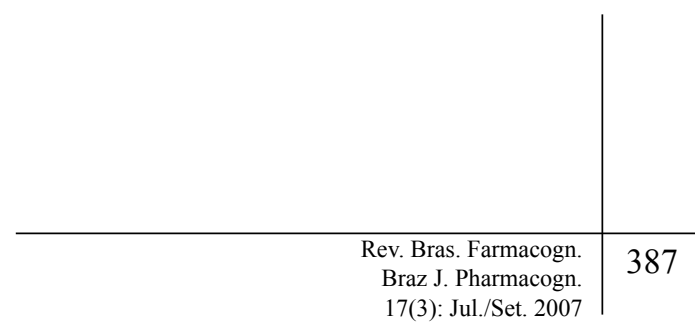

